\title{
Ensaios de sistemas de controle clássicos com uso de recursos didáticos significativos
}

João Alvarez Peixoto', Bruno Luiz de Pizzol²

' Laboratório de Automação Industrial. Universidade Estadual do Rio Grande do Sul (UERGS), Unidade de Porto Alegre, RS, Brasil. E-mail: joao-peixoto@uergs.edu.br

2 Laboratório de Automação Industrial. Universidade Estadual do Rio Grande do Sul (UERGS), Unidade de Porto Alegre, RS, Brasil. E-mail: bruno-pizzol@uergs.edu.br

\section{Resumo}

O aprendizado em sistemas de controle clássico é uma dificuldade nos cursos de engenharia. Os recursos computacionais amenizam o fato de serem analisados e equacionados por técnicas abstratas. Mas o maior efeito de um controlador é sua resposta ao distúrbio, porém este não pode ser simulado digitalmente devido sua característica aleatória. O objetivo da oficina de controle é a proposta de um aprendizado sobre controle de sistemas dinâmicos, a partir de recursos didáticos, que evidenciem os fenômenos físicos que ocorrem durante o processo de controle de forma lúdica. A metodologia do projeto prevê a pesquisa de requisitos dos sistemas, modelagem, implementação física, oficina de experimentos em controle ressaltando o maior efeito dos distúrbios. São implementados dois sistemas dinâmicos: sistema de controle de posição angular e de controle de servo posicionamento. Nestes sistemas os alunos modelam matematicamente, implementam controladores digitais e experimentam os resultados aplicando distúrbios para analisar a eficácia do controlador desenvolvido. É possível perceber que há uma grande diferença na qualidade da aprendizagem, quando esta acontece a partir de recursos didáticos significativos. As dificuldades no entendimento das teorias são minimizadas pela atração que os modelos físicos provocam. Os conceitos partem de uma abstração, o que requer maior esforço para compreender. É neste ponto que os recursos didáticos auxiliam na aprendizagem quando está trazendo os efeitos da abstração para realidade, permitindo correlação entre teoria e prática, proporcionando maior motivação por parte do aluno.

Palavras-chave: Controle digital. Sistemas dinâmicos. Técnicas de controle. Distúrbios. Aplicação de controle.

\section{Abstract}

\section{Trials of classical control systems using significant didactic resources}

Learning in classical control systems is a difficulty in engineering courses. The computational resources ease the fact that they are analyzed and equated by abstract techniques. But the major effect of a controller is its response to the disturbance, but this cannot be digitally-simulated because of its random characteristic. The objective of the control workshop is the proposal of a learning about control of dynamic systems, from didactic resources, that evidence the physical phenomena that occur during the process of control in a playful way. The methodology of the project foresees the research of systems requirements, modeling, physical implementation, control experiments workshop highlighting the effect of the disturbances. Two dynamic systems are implemented: angular position control system and servo positioning control. In these systems, students model mathematically, implement digital controllers, and test the results 
by applying disturbances to analyze the efficiency of the developed controller. Interest in the subject is also somewhat incremented, drawing more curiosity about physical / mechanical effects as they occur during the experiment. Difficulties in the understanding of theories are minimized by the attraction that the physical models provoke. Concepts depart from an abstraction, which requires greater effort to understand. It is at this point that didactic resources assist in learning, bringing the effects of abstraction to reality, allowing correlation between theory and practice, providing greater motivation on the part of the student.

Keywords: Digital control. Dynamic systems. Control techniques. Disturbance. Control application.

\section{Introdução}

O estudo de sistemas dinâmicos envolve a modelagem matemática, a análise e simulação de sistemas físicos de interesse da engenharia, como por exemplo, sistemas mecânicos, elétricos, hidráulicos, térmicos e assim por diante. No decorrer das disciplinas são apresentadas as formas de modelagem e técnicas de controle (OGATA, 2003). O objetivo do controle é manter uma variável física em um valor desejado, independente de distúrbios. Entretanto, a aplicação destas técnicas se dá em sua maioria através de aplicativos em software que simulam as condições da modelagem matemática, não simulando as peculiaridades de um sistema real. Isto significa que não conseguem simular distúrbios, o que na prática do ambiente industrial é muito comum.

$\mathrm{O}$ aprendizado de sistemas de controle tem sido algo que demanda um alto grau de esforço, face ao teor de abstração dos sistemas e técnicas de controle. Basicamente, o controle de um sistema dinâmico consiste em controlar a dinâmica do sistema através da interpretação do erro, oriundo da diferença entre o valor desejado e o valor atingido na variável de controle (DORF; BISHOP, 2009). E isto se dá por controladores, dispositivos que regra o comportamento do sistema dinâmico. A Figura I apresenta uma malha de controle típica.

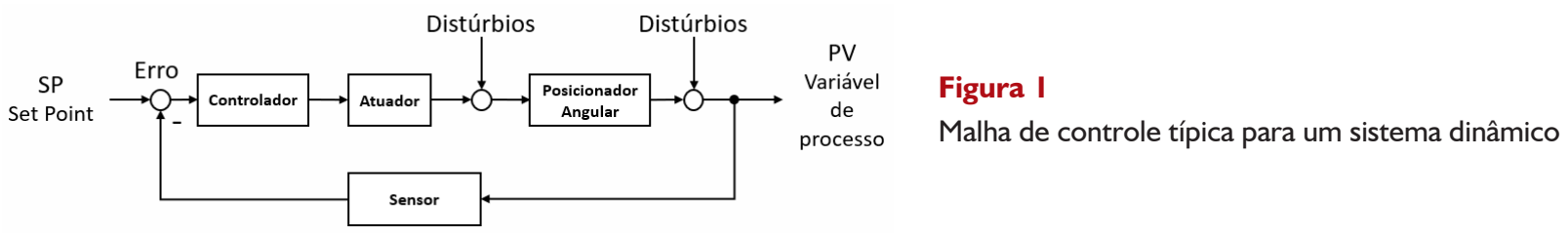

Fonte: Autores (2019).

O conhecimento se torna significativo quando o objeto de aprendizagem se torna evidente, de clara observação e pertinente ao contexto aplicado. Avaliar sistemas de controle com a possibilidade de perceber o significado dos distúrbios que afetam a planta torna a assimilação dos conceitos mais eficiente. Segundo Vygotsky (1998), os signos dão significado à aprendizagem, levando o aluno a associar conhecimento com ocorrência ou símbolos reais. Os recursos didáticos são os signos para que o aluno tenha o aprendizado dos sistemas de controle, quando inseridos em ambiente de ação de distúrbios.

A grande aplicabilidade destes recursos está no fato de servirem como uma plataforma para testes e demonstração de algumas das técnicas de controle, que poderão ser simuladas através dele, valendo-se do ambiente real como meio de ensaio, levando o aluno a observar os fenômenos da forma real como eles acontecem.

O objetivo deste trabalho é produzir recursos didáticos significativos para aprendizagem de sistemas de controle clássicos, validando-os através de oficinas de aprendizagem a alunos de cursos distintos, mas com necessidades de aprendizado de tais conceitos.

\section{Materiais e Métodos}

Uso de recursos didáticos significativos funciona como um facilitador no processo de aprendizagem. As plantas serão utilizadas como recursos didáticos para aplicação do conhecimento teórico adquirido nas disciplinas de controle.

A execução do projeto se estruturou no fluxo exibido na Figura 2, onde contam as etapas seguidas a cada passo do projeto. Nele se observa uma definição inicial dos modelos dinâmicos a serem estudados, sua implantação, seus ensaios na presença de distúrbios, sua validação em oficinas com alunos e a análise dos resultados alcançados, como balizador de ações tanto no ensino como na concepção de novos modelos. 


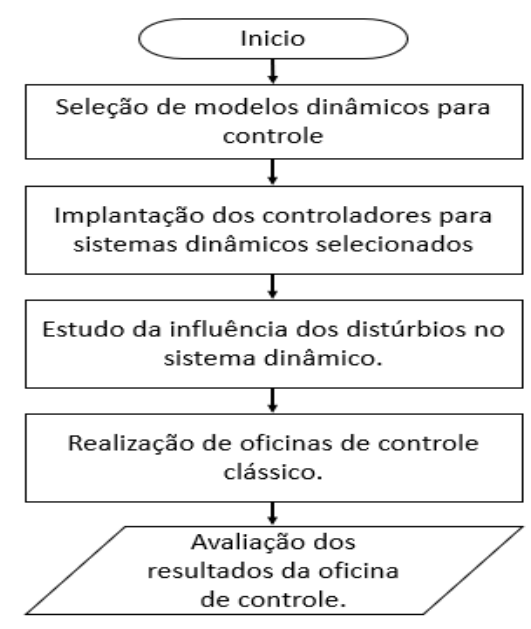

Figura 2

Fluxo da metodologia aplicada no projeto.

Fonte: Autores (2019).

De início tem-se 2 modelos a serem implementados como recursos didáticos. O primeiro deles, denominado como posicionador angular, visto na Figura 3, consiste em um conjunto de hélices divididas entre 2 motores de corrente continua fixados nas extremidades das asas e que através de um controle de rotação ajusta sua posição angular até atingir o setpoint escolhido pelo usuário, que pode variar de $0^{\circ}$ a $180^{\circ}$.

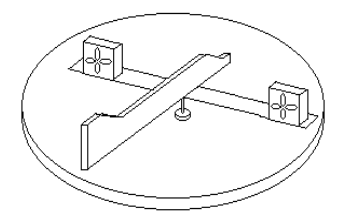

Figura 3

Esboço da planta do posicionador angular.

Fonte: Autores (2019).

A Figura 4 mostra o esboço da planta do servoposicionador, que através de sensores do tipo encoder cria-se um sistema de código binário, mais precisamente será utilizado código de Gray para interpretar as posições e também a direção de rotação.

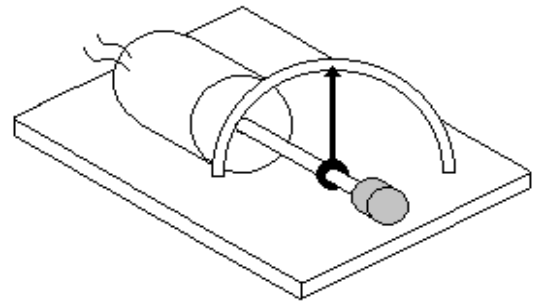

Figura 4

Esboço da planta do servoposicionador.

Fonte: Autores (2019).

Após a seleção, modelou-se matematicamente os dois experimentos, de forma a obter a malha de controle clássica, identificando a posição dos pólos, zeros e resposta ao degrau do sistema. A Figura 5 apresenta o modelo em diagrama de blocos do posicionador angular.

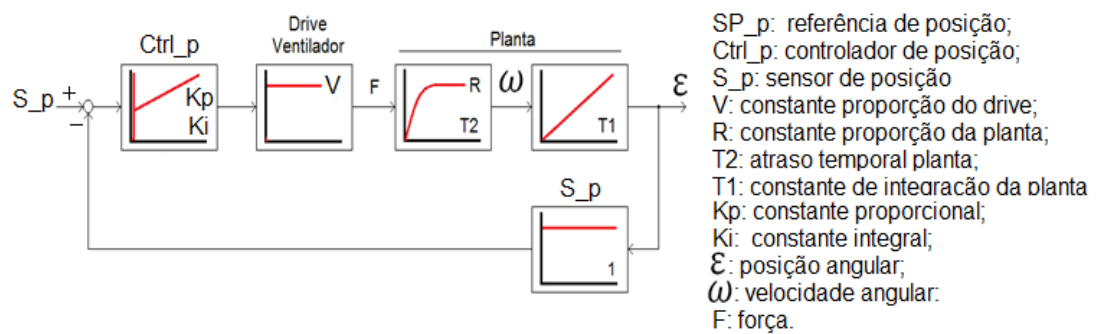

Figura 5

Modelo matemático do posicionador angular. 
Na Figura 6, é apresentado o modelo matemático de um controlador de temperatura.

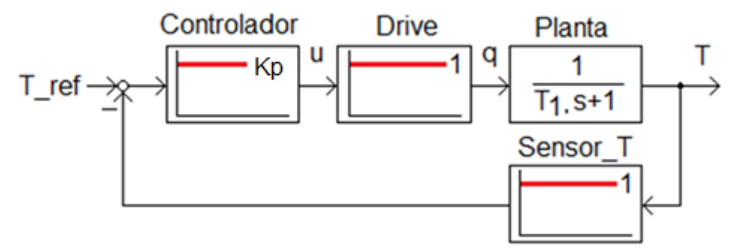

T_ref: temperatura de referência; Sensor_T: sensor de temperatura;
$\mathrm{Kp}$ : constante de proporcional; u: saida de controle;

q: energia calorifera;

Figura 6

T: temperatura

Modelo matemático do controlador de temperatura.

Fonte: Autores (2019).

Quanto à implementação do hardware e software, é utilizada uma placa de fenolite perfurada padrão para afixar os componentes eletrônicos, o microcontrolador do projeto é um Arduino Uno (MONK; LASCHUK, 2017), programado utilizando a linguagem $\mathrm{C}++$ através da IDE nativa do Arduino. A interface homem máquina conta com um display LCD 16 colunas por 2 linhas, com 4 botões que permite parametrizar os parâmetros de controle (NATALE, 200I). Na Figura 7, a seguir, se pode perceber a placa completa com o acionamento da planta, onde consta o posicionador angular, foi montado em material acrílico, com os motores de corrente contínua. $O$ sensor de posição é um potenciômetro limitado para rotacionar em $180^{\circ}$, gerando um sinal elétrico de 0 a $5 \mathrm{~V}$.
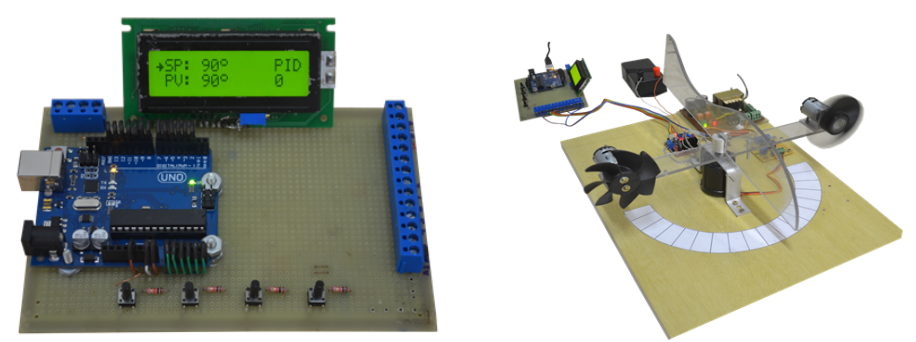

Figura 7

Circuito de controle e planta do servoposicionador

Fonte: Autores (2019).

E como fechamento do projeto, é proposto uma oficina de controle, onde alunos, egressos e comunidade acadêmica tem a oportunidade de vivenciar os efeitos do distúrbio sobre as plantas de sistemas dinâmicos.

\section{Resultados e Discussão}

O projeto se propôs em desenvolver uma oficina junto a dissentes, docentes e comunidade, em busca de investigar as formas com que o aprendizado do aluno em sistemas de controle ocorre, uma vez que há o uso de recursos didáticos significativos.

É proposto uma oficina de controle, em que inicialmente foram apresentadas as teorias e alguns conceitos de sistemas de controle SISO (single input single output), sistemas de uma entrada e uma saída, focando na experimentação prática e interagindo com o público a fim de relacionar os exemplos vistos na apresentação com os fenômenos do dia a dia de um estudante de engenharia. A proposta foi de que os alunos pudessem vivenciar como se parametriza o controle de uma planta, sob os efeitos de distúrbios, acompanhando o comportamento do controle clássico de sistemas dinâmicos em malha fechada. Após apresentada a teoria, foi dada uma explicação técnica de como funcionam os experimentos e como foi montado, tentando tornar a vivência mais interativa, e também para que o aluno possa entender de fato o que está acontecendo durante a experimentação e através de perguntas interativas para apontar no experimento aonde fica o controlador, atuador, sensor e planta.

Posteriormente, o projeto poderá ser aplicado nos componentes curriculares dos cursos de engenharia da universidade, assim como os recursos didáticos desenvolvidos para operacionalizar a oficina, que agora servem como recursos para tornar as aulas mais lúdicas.

Ocorreram duas oficinas de experimentação, em 8 de novembro de 2018 na unidade UERGS de Bento Gonçalves/RS e em 2I de novembro de 2018 na unidade UERGS de Novo Hamburgo/RS, totalizando um público total de 19 pessoas, alunos e pessoas da comunidade em geral, ligadas à área de engenharia. A Figura 8 mostra a oficina sendo ministrada. 


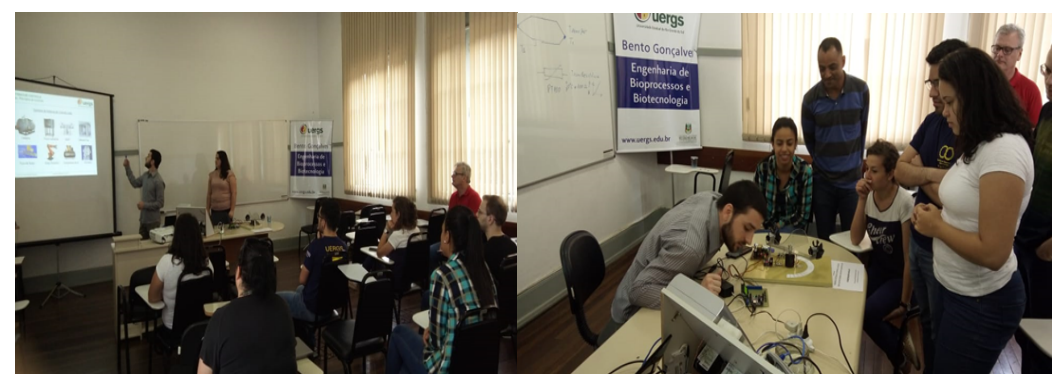

Figura 8

Oficina de controle sendo ministrada.

Fonte: Autores (2019).

Durante a oficina foi instigado aos alunos algumas tarefas, como por exemplo, trocar o setpoint, modificar os valores das constantes de proporção (kp), integração (ki) e derivação (kd), em uma malha de controle PID (proporcional-integral- derivativo), e lhes questionado quanto a correlação da teoria que foi apresentada com o experimento que está sendo realizado.

Para aferir o aprendizado dos alunos e a forma com que ele ocorreu, se utilizou um questionário na escala Likert (COSTA, 20I I), onde os alunos são instigados a responder segundo seu grau de concordância ou discordância sobre o assunto. É uma pesquisa em formato de oficina, de natureza aplicada ao ensino, classificada como pesquisa-ação, tendo foco os conceitos de sistemas de controle.

Ao todo foram 10 questões formuladas, sendo que 6 questões estavam relacionadas diretamente ao conteúdo, enquanto outras 3 delas estavam relacionadas com o aprendizado, sendo por fim I questão dissertativa que questionava quanto a satisfação do aluno com a oficina. Dentre as questões propostas, uma delas buscou analisar os recursos didáticos empregados na oficina e se eles ajudaram o aluno a entender $e$ compreender o conteúdo e sua aplicação e propósito para a disciplina.

As questões propostas aos alunos ao final da oficina foram as seguintes:

I. Uma malha fechada de controle clássico de sistemas dinâmicos leva em consideração a ação da planta, dos atuadores, do sensoriamento e do controlador, sendo os demais fatores irrelevantes para este modelo;

2. Ao definir o modelo matemático de um sistema dinâmico, deve levar em consideração as variações que a planta pode ter ao longo do tempo, bem como os distúrbios que podem afetar o sistema;

3. Uma malha de controle pode ser simulada em software, bastando inserir os modelos do sistema dinâmico, do sensoriamento e do controlador, onde a resposta ao degrau irá fornecer o comportamento real do sistema controlado, inclusive sua reação aos distúrbios que se fizerem presentes;

4. A função de um controlador em uma malha fechada de controle clássico de sistemas dinâmico é manter a variável de processo no valor desejado como referência, mesmo que haja atuação de distúrbios, mesmo afetando as condições da planta;

5. Para conhecer o efeito de um distúrbio, basta monitorar a equação característica da função de transferência do sistema dinâmico, que representa tal efeito através de seu diagrama de localização de pólos e zeros no plano S;

6. Um sistema de controle digital (microntrolador) pode ser considerado um sistema de controle linear, uma vez que a velocidade de ação do controlador (sensoriamento, definição do erro e atuação de controle) seja muito maior do que o sistema dinâmico a ser controlado, podendo ser aplicada as técnicas de análise e controles clássicos lineares;

7. Os recursos didáticos te ajudaram a entender e compreender o conteúdo e sua aplicação e propósito para a disciplina;

8. Os recursos didáticos utilizados facilitaram a sua aprendizagem;

9. Você se sentiu mais motivado/ envolvido a aprender com o uso dos experimentos;

10. Por fim, deixe aqui seu relato sobre a oficina de controle e instrumentação.

A Tabela I apresenta o questionário aplicado e seu percentual de resposta dos 19 alunos respondentes. 


\begin{tabular}{c|c|c|c|c|c|c}
\hline Questão & Resposta esperada & Concordo plenamente & Concordo & Não sei opinar & Discordo & Discordo plenamente \\
\hline 1 & Discordo & $5 \%$ & $42 \%$ & $16 \%$ & $21 \%$ & $16 \%$ \\
\hline 2 & Concordo & $58 \%$ & $42 \%$ & $0 \%$ & $0 \%$ & $0 \%$ \\
\hline 3 & Discordo & $5 \%$ & $58 \%$ & $16 \%$ & $21 \%$ & $0 \%$ \\
\hline 4 & Concordo & $37 \%$ & $53 \%$ & $0 \%$ & $11 \%$ & $0 \%$ \\
\hline 5 & Discordo & $11 \%$ & $53 \%$ & $16 \%$ & $21 \%$ & $0 \%$ \\
\hline 6 & Não sei Opinar & $5 \%$ & $26 \%$ & $32 \%$ & $37 \%$ & $0 \%$ \\
\hline 7 & Concordo & $79 \%$ & $21 \%$ & $0 \%$ & $0 \%$ & $0 \%$ \\
\hline 8 & Concordo & $95 \%$ & $5 \%$ & $0 \%$ & $0 \%$ & $0 \%$ \\
\hline 9 & Concordo & $74 \%$ & $26 \%$ & $0 \%$ & $0 \%$ & $0 \%$ \\
\hline
\end{tabular}

Fonte: Autores (2018).

Analisando a questão I, o esperado era uma discordância, mas não ocorreu, fato que leva a repensar a forma com que a teoria foi abordada, no que diz respeito aos elementos que compõe uma planta dinâmica e as variáveis que afetam. Assim como na questão 3 , onde não ficou claro a ineficiência dos aplicativos em software para realizar tais simulações.

Já a questão 5 gerou uma dúvida quanto ao tópico, face a distribuição com que as opiniões se apresentaram. Este tópico deverá ser mais enfatizado em futuras edições desta oficina.

A questão 6 é uma forma de validar o instrumento que está sendo aplicado (questionário), pois foi proposto um assunto que não foi abordado na oficina, nem em sua etapa teórica de apresentação de conceitos, nem na sua parte prática no uso dos recursos didáticos. O resultado comprova a eficácia do instrumento, onde o aluno realmente está respondendo segundo sua conviç̧ão.

As questões 7, 8 e 9 comprovam a hipótese deste trabalho, apontando que os recursos didáticos significativos realmente facilitam a aprendizagem.

A questão 10 solicitou que os alunos manifestassem sua opinião quanto à oficina realizada, tendo as seguintes respostas como depoimentos dos alunos:

"A oficina me deixou bastante animado com a próxima disciplina do curso, visto que ainda estou no segundo semestre. As explicações foram claras e interessantes. "

"Foi uma troca de experiências muito boa e que facilitaram o entendimento de alguns termos porque na teoria não fazia muito sentido."

"Gostei muito da oficina, a didática foi ótima e os recursos usados ajudaram a entender o que ocorre na prática o que aprendemos na teoria. “

"Achei muito didática a oficina, mesmo no começo do curso entende bastante. “

"Foi muito bom porque deu para ver a prática o que as vezes a gente desconfia quando resolve os cálculos. A oficina foi mais proveitosa do que esperava. "

"Obteve-se um rendimento excepcional no processo ensino-aprendizagem. “

"Muito boa! Parabéns ao professor e alunos bolsistas pela disposição, conhecimento passado e paciência. “

"Oficina foi fundamental para entender como os dispositivos interagem para atingirem o objetivo desejado no processo."

"A oficina foi muito proveitosa. O ministrante apresentou uma abordagem muito didática e objetiva. “

Aqui se pode comprovar as teorias que fundamentam o aprendizado significativo. Segundo Ausubel (1978), o importante para comprovar a efetividade pedagógica, pois com a teoria da aprendizagem significativa, é possível estabelecer organização nos novos conhecimentos na estrutura cognitiva do aluno. Esses novos conhecimentos foram agregados à estrutura cognitiva já existente, observa-se através dos relatos que os alunos puderam compreender os dois recursos didáticos, puderam visualizar os distúrbios ocorridos, conseguiram associar e fortalecer conteúdos e conceitos já existentes ao relacionar e visualizar a aplicação de conteúdos de outras disciplinas do curso, o que demostra possibilidade da confluência de conteúdos e informações aplicadas em experimentos didáticos.

Segundo Vygotsky (1998), os signos dão significado à aprendizagem, levando o aluno a associar conhe- 
cimento com ocorrência ou símbolos reais. Os recursos didáticos são os signos para que o aluno tenha $\circ$ aprendizado dos sistemas de controle, quando inseridos em ambiente de ação de distúrbios.

A pesquisa científica dentro da proposta de realização da oficina de controle foi apresentada no $8^{\circ}$ SIEPEX, em Cachoeira do Sul - RS (PIZZOL; KLEIN; PEIXOTO, 20I8), onde foram discutidos os aspectos técnicos da implementação dos recursos didáticos, os aspectos pedagógicos da aplicação de tais recursos e a ciência, que diz respeito à pesquisa de aplicações par ao tópico abordado.

\section{Conclusão}

A aprendizagem em sistemas de controle se torna dificultada pelo fato de que não se consegue visualizar os efeitos de um distúrbio atuando sobre a planta e os conceitos partem de uma abstração complexa, o que requer maior esforço para melhor entendimento, é neste ponto que os recursos didáticos significativos entram auxiliando na aprendizagem. Com base nas respostas anteriores, é possível perceber uma grande diferença na qualidade de aprendizagem quando recursos didáticos significativos são utilizados como apoio pedagógico, pois trazem os efeitos da abstração para realidade, apresentando seus efeitos no momento e na magnitude que eles ocorrem, permitindo correlação entre teoria e prática, proporcionando maior motivação para o aprendizado do aluno.

Já se pode vislumbrar que no ensino de engenharia os recursos didáticos propostos neste projeto serão usados como apoio na aprendizagem significativa, fato que comprova ao analisar os resultados obtidos nas oficinas, assim como, formação de novos conceitos bem como o fortalecimento dos já apreendidos e organização da estrutura cognitiva sem alterar seu significado.

\section{Referências}

AUSUBEL, D. P. Psicologia Educativa: um punto de vista cognoscitivo. México: Trilhas, 1978.

COSTA, F. J. Mensuração e desenvolvimento de escalas: aplicações em administração. Rio de Janeiro: Ciência Moderna, 20II.

DORF, R. C.; BISHOP, R. H. Sistemas de Controle Modernos. I I. ed. Rio de Janeiro: LCT, 2009.

MONK, S.; LASCHUK, A. Programação com Arduino: Começando com Sketches. 2. ed. São Paulo: Bookman, 2017.

NATALE, F. Automação industrial. 3. ed. São Paulo: Érica, 200 I.

OGATA, K. Engenharia de Controle Moderno. ed. 4. [s.I]: Prentice-Hall, 2003.

PIZZOL, B. L. De; KLEIN, V. L.; PEIXOTO, J. A. Ensaios de sistemas de controle clássicos com uso de recursos didáticos significativos. In: VIII SIEPEX, UERGS, 2018, Cachoeira do Sul. Anais eletrônicos.... Disponível em: https://www. doity.com.br/anais/8-siepex/trabalho/58224. Acesso em: 12 de dez. 2018.

VIGOTSKY, L. S. Pensamento e Linguagem. São Paulo: Martins fontes, 1998. 